\title{
Human Body Measurements and Clothing Virtual for Men Adult Consumers
}

\author{
Abdul Kareem A. Najem Al-Aloosy \\ Anbar University \\ College of CS, Iraq
}

\author{
Muzhir Shaban Al-Ani \\ Anbar University \\ College of CS, Iraq
}

\begin{abstract}
There are many differences in human size and some of these sizes are abnormal. The purpose of this approach is to locate and measures the specific dimensions of human body in order to choose the fitted dress. This approach is concentrated on men adult consumer to be more specific system used for men plant or men shopping center. There are many standard sizes for human body and these sizes differ from country to other. So this approach try to find a specific standard for human body depends on his overall dimensions comparing with the standard sizes. The proposed system depends on two cameras and simple processing algorithms to predicate with the adequate size.
\end{abstract}

\section{General Terms}

Human Body Measurements, Clothing Virtual Men Adult Clothing, Human Body Clothing et. al.

\section{Keywords}

Body Measurements, Size Measurements, Cloths Fitting, and Clothing Fit Information.

\section{INTRODUCTION}

Nature has huge number of symmetrical patterns. One of the most important examples of symmetrical pattern is the human body. Phi (1.618) is the Golden Number, is a proportion that found in many areas of the nature as well as in the structure of the human body. Many of the bones that form our skeleton are thought to have a proportional relationship of 1:1.618 [1].

Cloths industries try to produce products according to the customer requirements. Some of these requirements concentrated on cost, quality and dimensions. This paper will be concentrated on the cloths dimensions that are very important to the customers because of the big differences between the existed standards [2].

In this paper we try to fit suitable cloths for each person according to the standard size. Considering that there are abnormal human sizes, which are special sizes.

\section{STANDARD SIZE}

There are many standard for human size cloths. Table (1) shows the standards of human size cloths for different countries [3].

Table (1) standard of human size cloths

\begin{tabular}{|l|l|l|l|l|l|l|l|l|l|}
\hline Size number & $\mathbf{1}$ & $\mathbf{2}$ & $\mathbf{3}$ & $\mathbf{4}$ & $\mathbf{5}$ & $\mathbf{6}$ & $\mathbf{7}$ & $\mathbf{8}$ & $\mathbf{9}$ \\
\hline USA & 32 & 34 & 36 & 38 & 40 & 42 & 44 & 46 & 48 \\
\hline UK & 32 & 34 & 36 & 38 & 40 & 42 & 44 & 46 & 48 \\
\hline Australia & 32 & 34 & 36 & 38 & 40 & 42 & 44 & 46 & 48 \\
\hline $\begin{array}{l}\text { Europe } \\
\text { Continental }\end{array}$ & 42 & 44 & 46 & 48 & 50 & 52 & 54 & 46 & 58 \\
\hline Japan & $\mathrm{S}$ & $\mathrm{S}$ & $\mathrm{S}$ & $\mathbf{M}$ & $\mathrm{L}$ & L & LL & LL & LL \\
\hline France & 42 & 44 & 46 & 48 & 50 & 52 & 54 & 46 & 58 \\
\hline Russia & 42 & 44 & 46 & 48 & 50 & 52 & 54 & 46 & 58 \\
\hline $\begin{array}{l}\text { Characters } \\
\text { size }\end{array}$ & $\mathbf{X S}$ & $\mathbf{S}$ & $\mathbf{S}$ & $\mathbf{M}$ & $\mathbf{M}$ & $\mathbf{L}$ & $\mathbf{X L}$ & $\mathbf{X X L}$ & XXXL \\
\hline Neck & 33 & 36 & 37 & 38 & 39 & 41 & 43 & 46 & 48 \\
\hline Chest & 84 & 89 & 94 & 102 & 107 & 112 & 122 & 132 & 142 \\
\hline Arm & 81 & 83 & 84 & 86 & 89 & 90 & 91 & 93 & 94 \\
\hline Waist & 71 & 74 & 79 & 84 & 89 & 94 & 104 & 137 & 132 \\
\hline
\end{tabular}




\section{SPECIFIC HUMAN DIMENSION DEFINITIONS}

As we mentioned that this approach will be valid form men adult so the men human adult body can be divided into two parts and each part can be carry many dimensions as below [4]:

- Top dimensions, which included:

1. Waist; measure around the natural waistline.

2. Inseam; measure from the crotch seam to the bottom of the leg.

- Bottom dimensions that included:

1. Neck; measure around the middle of the neck.

2. Shoulders; measure between the two shoulders.

3. Chest; measure under the arms around the fullest part of the chest.

4. Waist; measure around the natural waistline.

5. Arm length; measure across the shoulder to the elbow and down to the wrist.

\section{RELATED WORKS}

Many works are published in this field, below some of these works:

Nadia Magnenat-Thalmann, Hyewon Seo (2004), Frederic Cordier (2004) studied the automatic modeling of animatable virtual humans and proposed their problems and solutions. This paper studied the capturing method via the shape of real people, and then generates parameters for modeling static shape to indicate the variety of human body shapes. In addition a dynamic shape is captured via the changes of human body shape via their moves. Also virtual humans are captured classified, summarized and compared. Finally, the tested methods for clothed virtual humans are explained and reviewed [5].

Yue-Qi Zhong (2008), proposed an efficient method to accelerate the dress-up humans procedure. Firstly, the virtual garment is dressed using a human model in a standing pose to capture a distance map between the garment shell and the human body model. Then the distance map is used to generate a coarse match when the pose of the human body model has been changed. Finally, generate an efficient 3D configuration without surface penetration after a draping/relaxation scheme. The obtained results indicated that this method is a useful and fast treatment for dressing sewn virtual garment on various posed human body models [6]

Yinpeng Chen, Zicheng Liu and Zhengyou Zhang (2013), presented an efficient method to model 3D human body including the variations on both human shape and pose, by exploring a tensor decomposition technique. It is clear that 3D human body modeling is important for 3D reconstruction and animation of realistic human body, which can be widely used in many applications such as Tele-presence and video game applications. The tensor-based model addresses this issue by jointly modeling shape and pose deformations [7].

Md. Zahangir Alom, Farazul Haque Bhuiyan and Hyo Jong Lee (2013), proposed a system consists of multiple tasks including extraction of different body parts, torso detection, resizing input dress images and dress up using blending and reblending techniques over the subject. The coexistence of different clothing and cluttering backgrounds is the main difficulty for accurate body extraction in image. Haar classifier is applied for detecting face from input frames and geometrical information is used to extract different parts (like a face, a torso, and hands) of a body according to the face position in a frame. Due to the variability of human body, it is complicated to extract accurately [8].

Yueh-Ling Lin and Mao-Jiun J. Wang (2014) proposed a method to evaluate clothing fit on human body by dressing virtual clothes on 3D human model. In this paper several items were tested to ensure the system. In order to evaluate the clothing fit for virtual try-on, the representations of clothes with different size are compared with virtual and human fit trials using visual analysis. For objective fit evaluation, the amount of clothing fit of specified size is assessed quantitatively by measuring the area of vacant space between clothes and human body. Finally, the clothing fit information can be applied for the development of clothing products in apparel industry [9].

\section{THE PROPOSED APPROACH}

The proposed approach can be implemented in a shopping center in which the size of the customer can be predicted at the time that the customer enter the first gate of the shopping center. This system contains the following devices as shown in figure (1):

- Sensors that are fixed at the entering gate for giving a signal to start capturing the camera.

- Two cameras are fixed in front and behind the entering gate.

- Central processing unit that process the required operation.

- Grabbing device to store the required data.

- Screen display to see the fitted size and dress operations.

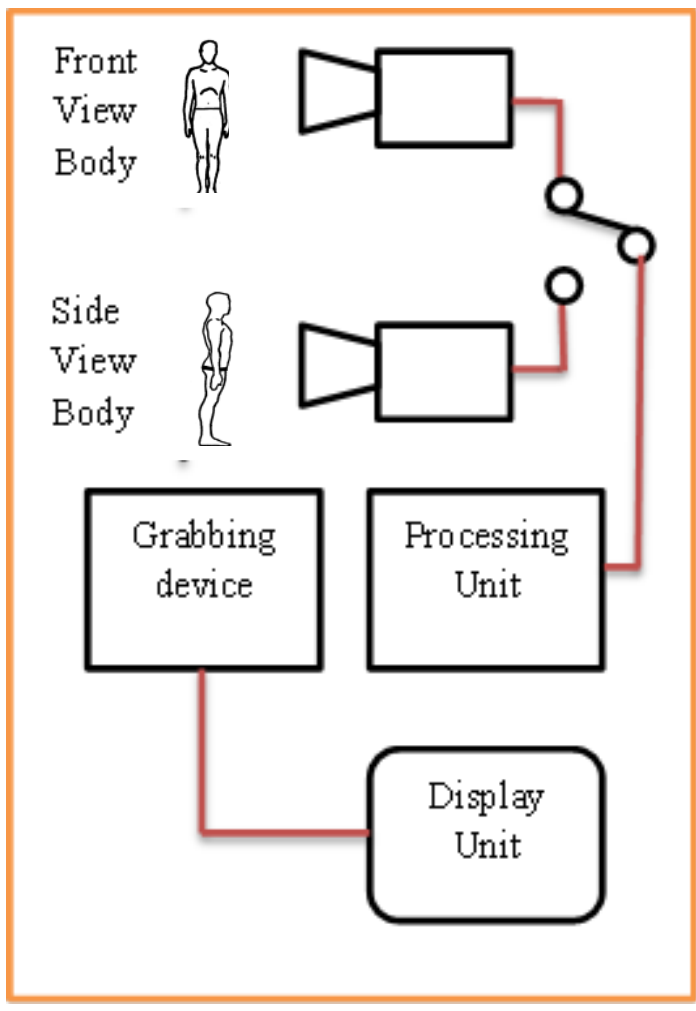

Figure (1) components of the proposed system 
The operation of the proposed system can be divided into many steps as shown in figure (2).

- Step1: image acquisition that deals with the front and side views images. This is implemented via the two fixed cameras.

- Step2: calculate the required dimensions that deals with top and bottom dimensions. This is calculated via the implemented algorithms.

- Step3: Compare the result that deals with the comparison of the obtained dimensions with the standard sizes. This is calculated via the implemented algorithms.

- Steps4: size prediction that deals with predicted the adequate size for the customer. This is implemented via a lookup table of the standard sizes.
The image processing approach as shown in figure (3) including the following:

- Image acquisition for both directions.

- Extract 2D human body shape clothing for both directions.

- $\quad$ Estimate 2D human body shape under clothing for both directions.

- Calculate the required factors of the estimated 2D human body shape under clothing.

- Compare these factors with the standard sizes.

- $\quad$ Predicate the suitable standard size.

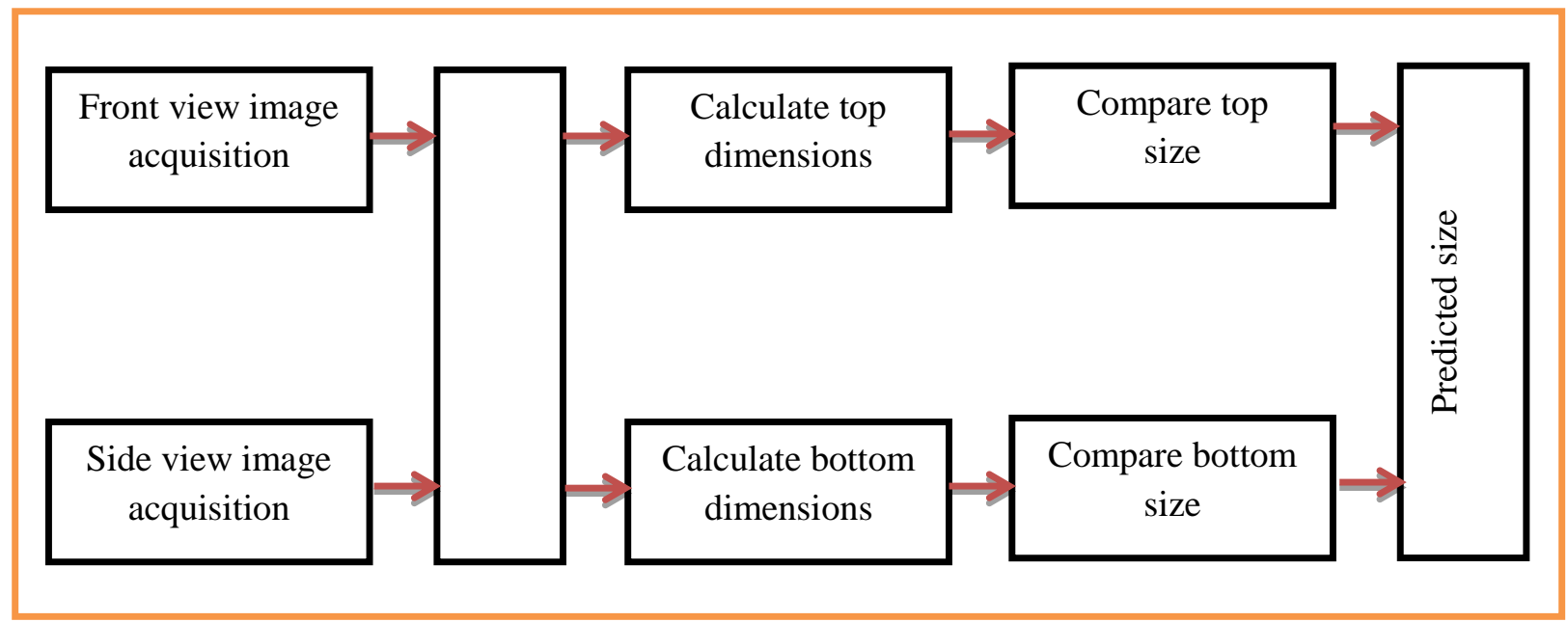

Figure (2) Processing steps

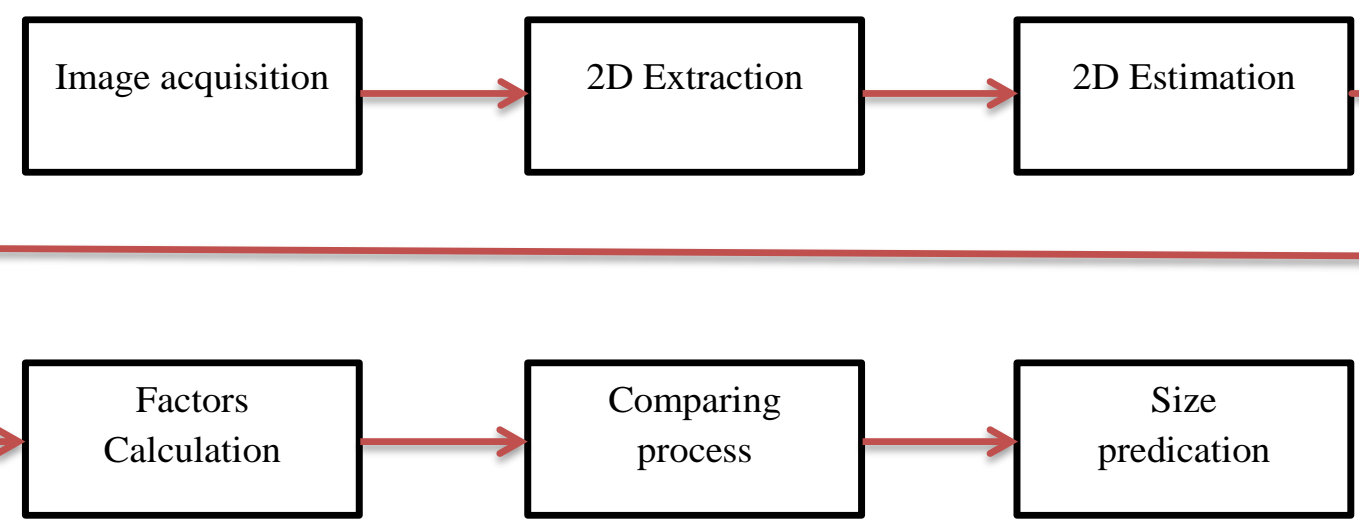

Figure (3) image processing approach 


\section{RESULTS AND ANALYSIS}

The proposed system is implemented and simulated via Matlab package programming language. When the two images (front view and side view) are acquired and introduced via the implemented system, then the processing steps will be started according to the order. The processing steps passing from converting images into gray scale until reaching the final decision as denoted in the following:

- Converting these two received images into gray scale images as shown in figure (4).
- $\quad$ Filtering the gray scale images with adaptive filter as shown in figure (5).

- Applying edge detection process of the adaptive images as shown in figure (5).

- Applying histogram of gray scale images as shown in figure (6).

- Applying histogram of equalized gray scale images as shown in figure (7).

- Calculate the requited dimensions according to figure (8).

- Compare the obtained dimensions with the standard dimensions.

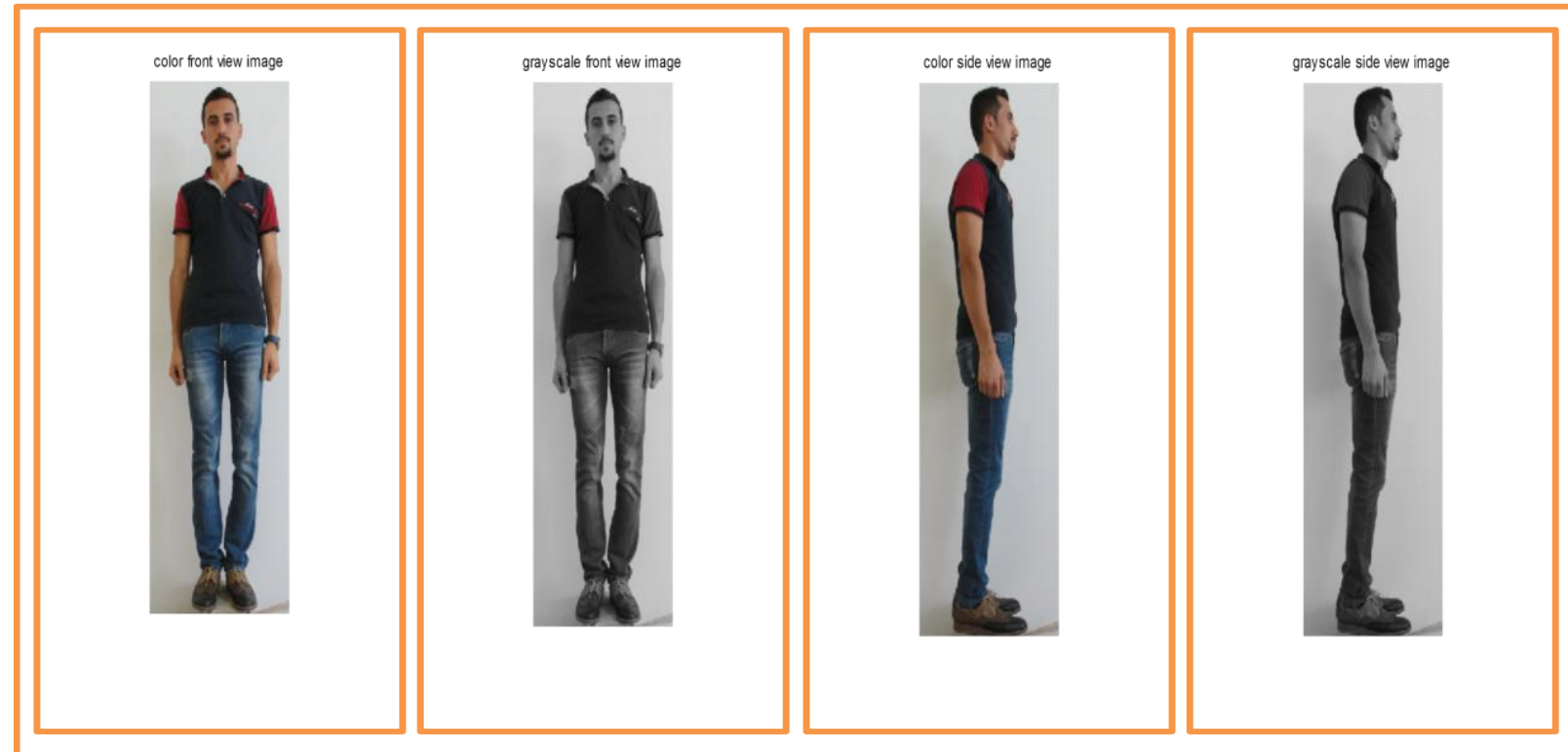

Figure (4) color and gray scale images of front and side views

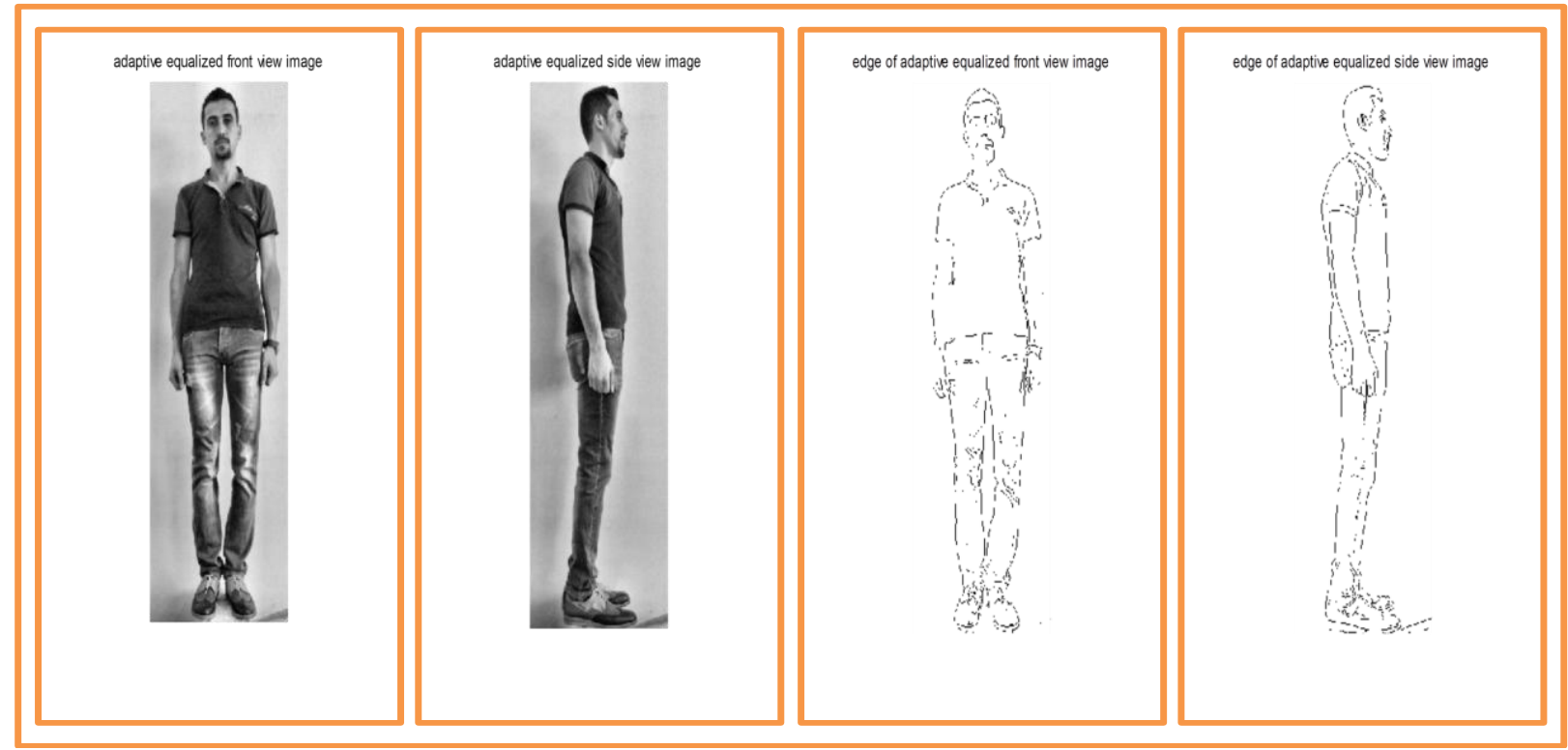

Figure (5) adaptive and edge images of front and side views 


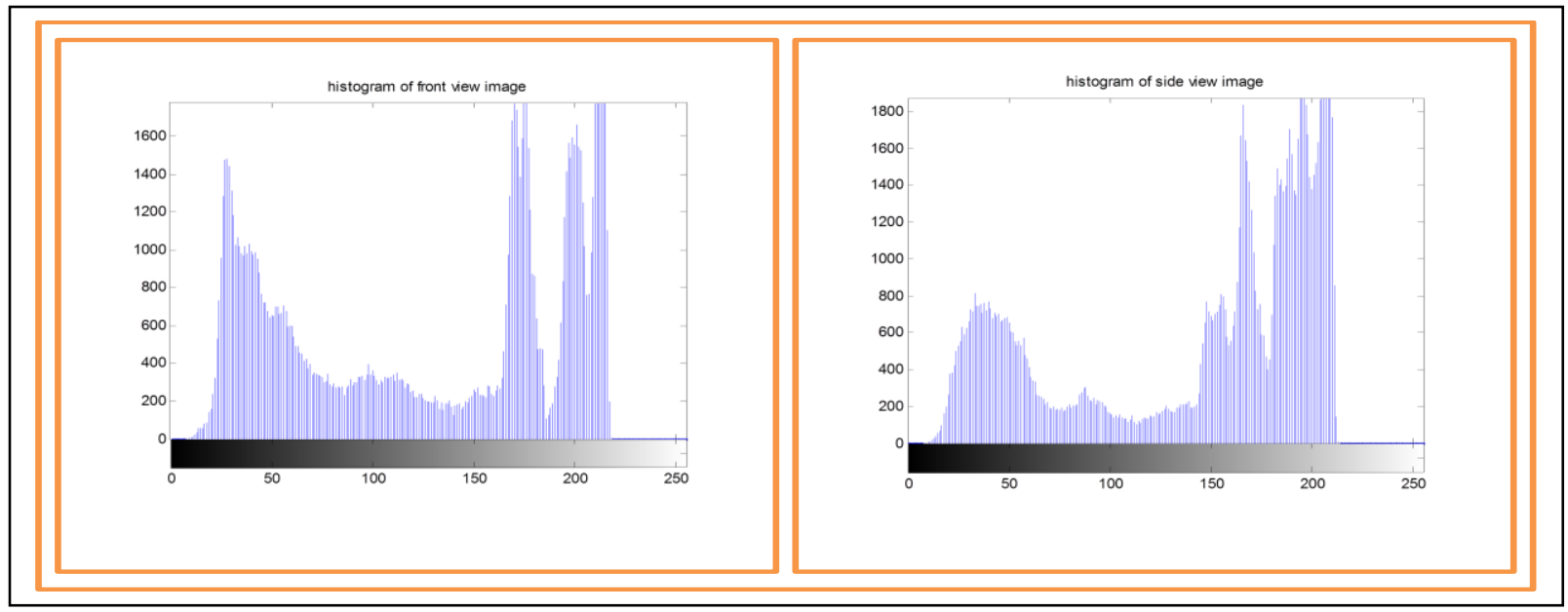

Figure (6) histogram of gray scale of front and side views images

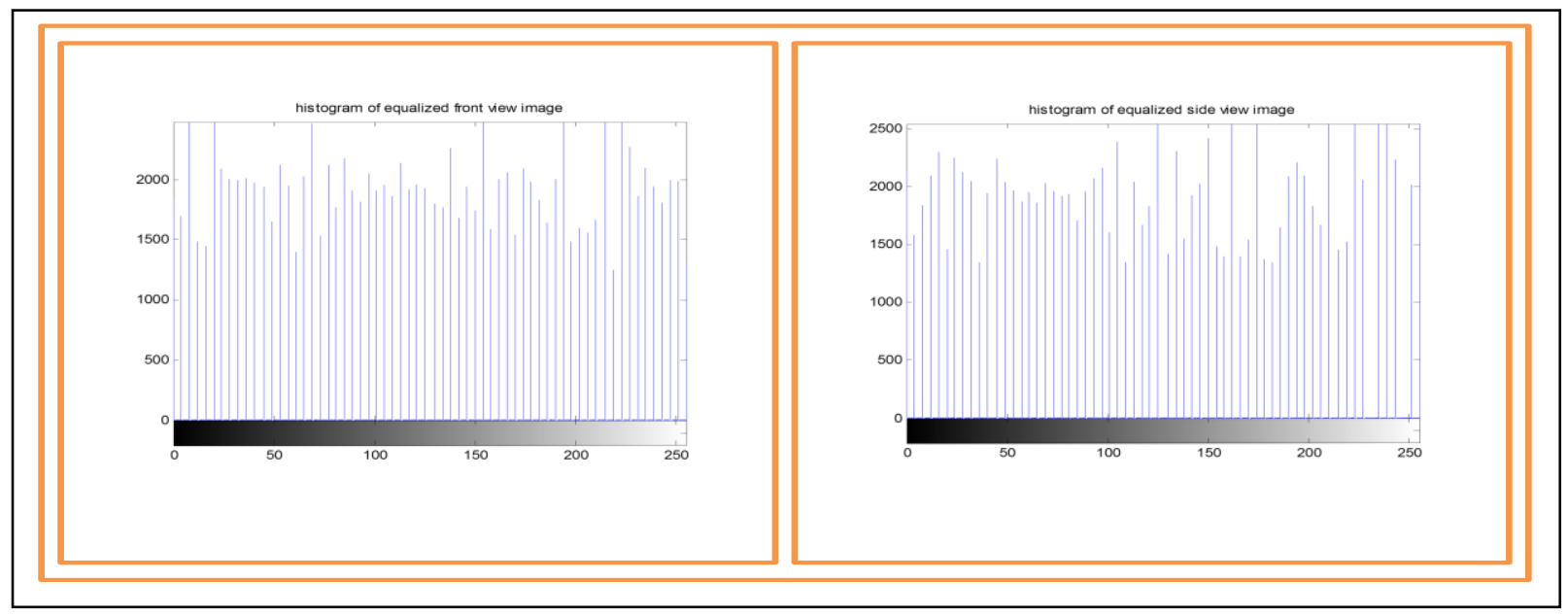

Figure (7) histogram of equalized gray scale of front and side views images

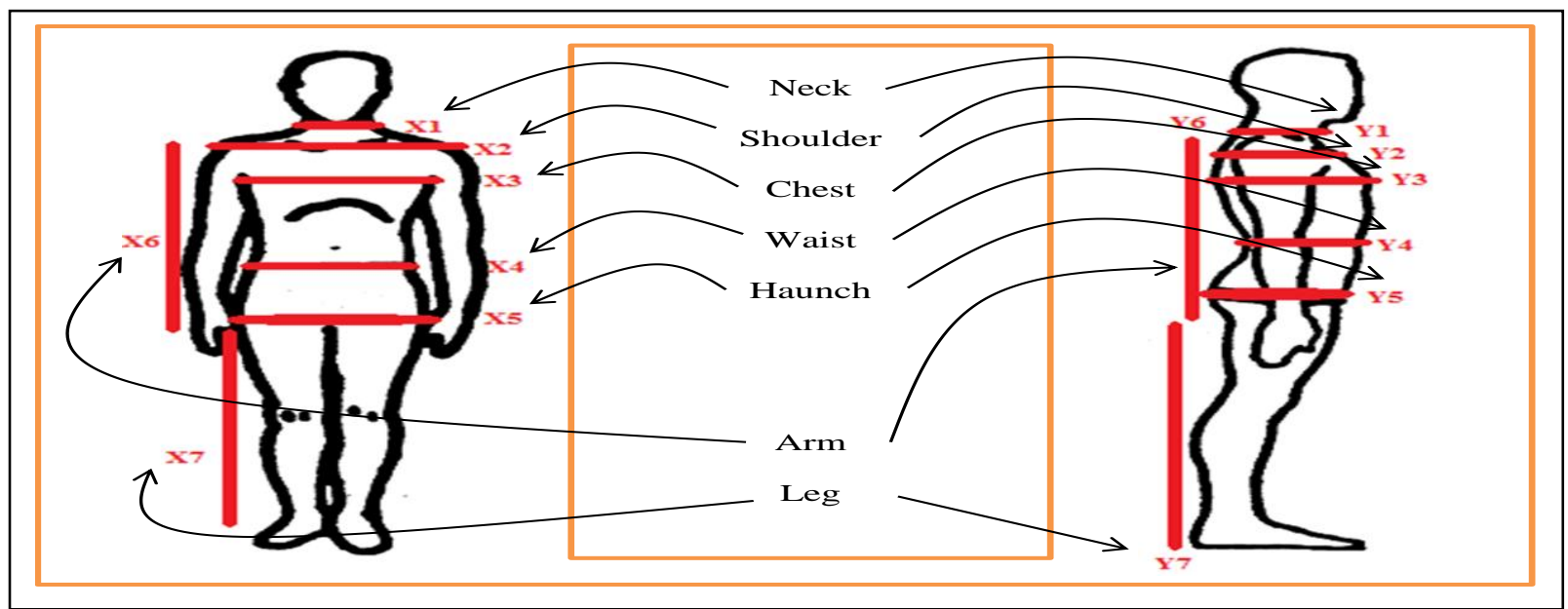

Figure (8) Calculation of dimensions of front and side views 


\section{CONCLUSIONS}

The implemented system presents an approach for evaluating the human body dimensions in order to predict the adequate size. This system aims to predicate the acceptable size for a person when he passed through the shop gate. The predication of size depends on image processing approach in which mapping these sizes into standard sizes. The processing steps pass via matching the two images (front view and side view) to generate the required measures. There are no problems with standard sizes of human body but the problems arise with the abnormal sizes. These problems can be reduced as possible with the predicted approximation.

\section{REFERENCES}

[1] Alvin R. Tilley, Henry Dreyfuss Associates, "The Measure of Man and Woman: Human Factors in Design Revised Edition”, Wiley New York, 2002.

[2] ISO 7250-1:2008, "Basic human body measurements for technological design -- Part 1: Body measurement definitions and landmarks", 2008.

[3] Julius Panero and Martin Zelnik, "Human Dimension \& Interior Space: A Source Book of Design Reference Standards", USA, 1979.
[4] Tom Flynn, "The Body in Three Dimensions", Kalmnn an King Publisher, New York 1998.

[5] Nadia Magnenat-Thalmann, Hyewon Seo, Frederic Cordier, "Automatic Modeling of Virtual Humans and Body Clothing", Journal of computer science and technology, vol.19, no.5, pp575-584, 2004.

[6] Yue-Qi Zhong, "Fast Virtual Garment Dressing on Posed Human Model", Journal of Fiber Bioengineering and Informatics, JFBI Vol.1 No. 12008.

[7] Yinpeng Chen, Zicheng Liu and Zhengyou Zhang, "Tensor-Based Human Body Modeling", CVPR2013 conference, 2013.

[8] Md. Zahangir Alom, Farazul Haque Bhuiyan and Hyo Jong Lee, implemented Real Time Dress-up System based on Image Blending, International Journal of Computer Applications (0975 - 8887) Volume 75No.1, August 2013.

[9] Yueh-Ling Lin and Mao-Jiun J. Wang, "Digital Human Modeling and Clothing Virtual Try-on", Proceedings of the 2014 International Conference on Industrial Engineering and Operations Management Bali, Indonesia, January $7-9,2014$. 